\title{
Same-Fretted-Note Intonation Variability of the Steel String Acoustic Guitar
}

\author{
R.M. MOTTOLA ${ }^{1}$
}

Abstract- Ten experienced guitar player subjects were recruited to play twenty notes each at fret positions 1, 5 and 12 on the $G$ string of a typical steel string acoustic guitar with typical string set and setup. The notes were digitally recorded and analyzed for pitch errors. Summary statistics of the pitch errors were calculated for each note and for each note/subject. Data was displayed graphically for visual examination. Results indicate variability across subjects and across notes tested. Results for the entire subject population show pitch variability of \pm 2 cents from mean value for each of the three notes at one standard deviation from mean, indicating variability of \pm 2 cents from mean value for $\approx 68 \%$ of samples and of \pm 4 cents from mean value for $\approx 95 \%$ of samples.

\section{INTRODUCTION}

Achieving pitch accuracy of fretted notes of a fretted stringed instrument is a complex problem. Although guitar fret placement typically follows the frequency relationship of the pitches of the equal temperament scale, intonation errors are introduced by the process of fretting each note as the string must be stretched to press it down to the fret. Intonation errors are also caused by bending stiffness of the string, which adds an additional restoring force to the vibrating string. For these reasons most guitars employ some modification to the nut/frets/bridge saddle geometry, intended to improve overall intonation accuracy. The term used to describe all such compensatory modifications is compensation. The most common compensation technique is to lengthen the vibrating length of the strings by moving the location of the bridge saddle farther away from the nut. This lengthening flattens the pitch and thus compensates for the sharping effects of string stretch and stiffness during fretting. In practice the lengthening is performed empirically so that the pitch of the fretted $12^{\text {th }}$ fret is an octave above that of the open string. Mathematical modeling of guitar intonation with bridge compensation indicates that this technique improves overall intonation but can provide ideal intonation only for the open string and the octave at the $12^{\text {th }}$ fret.

There has been considerable interest in improving the intonation of fretted notes of the guitar is recent years. Much of the research in this area appears in guitar construction literature. Much of the early research involved the classical guitar[1-3]. In addition to bridge saddle compensation, these works explore the effects of adding nut compensation (moving the nut location relative to the frets). One of these works[1] proposes additional accuracy benefits achievable by relocating the positions of frets. Later researchers looked at this issue as it relates to the steel string acoustic guitar[4-7]. Although the physics are the same for both instruments, intonation errors associated with fretting tend to be greater for steel string guitars due to the higher stiffness of steel strings compared to the nylon strings of the classical guitar. Proposals for intonation improvement in these latter works include additional nut compensation and some also propose changes to fret placement. Note that there exist commercial products marketed to improve guitar intonation including replacement nuts and fretboards with altered fret locations, but none are specifically referenced here because mathematical descriptions of them are lacking. In addition to its proposed use of bridge saddle and nut compensation, [7] is interesting and unique among the works cited for two reasons. The first is that it compares experimental intonation results to a measure of human pitch sensitivity[8] and so proposes a practical lower limit to the requirement of pitch accuracy. This work inspired a recent research effort on human perception of intonation differences among various steel string guitar compensation strategies commonly in use[9]. The second unique and interesting thing in [7] is that the paper describes the results of physical testing of its presented mathematical compensation model. Physical testing was performed using a modified sonometer. Results indicate considerable variability in pitch for the same string/fret combination, probably due to variable fretting pressure and inadvertent string bending[10], i.e. lateral displacement of the string during fretting. It is not clear from that study if the extent of pitch variability measured on the sonometer would also be seen on the guitar.

\footnotetext{
${ }^{1}$ R.M. Mottola - Liutaio Mottola Stringed Instrument Design, Newton MA 02460 USA, rmm at LiutaioMottola dot com
} 
Documenting same-fretted-note pitch variability of the steel string acoustic guitar would clearly be a useful exercise and is the subject of this study. Each time a player plays a note at the same string/fret position the pitch of that note will be different. The extent of that difference for each string/fret position and for each player represents uncontrollable variability in the pitch accuracy of the instrument. This variability is clearly an important limit on the accuracy and effectiveness of any system intended to improve intonation. At the very least, variability will impose lower limits to the effectiveness of any intonation improvement strategy.

\section{DESCRIPTION OF THE EXPERIMENT}

Eleven experienced guitarists (mean number of years of guitar playing experience $=42$ ) were recruited at a meeting of guitar luthiers to take part in the experiment. Data from one of these subjects was omitted from subsequent analysis due to the presence of substantial vibrato (purposeful pitch variation) in the recorded samples from that subject. All guitarists played a series of notes at three string/fret locations on the same guitar in a quiet room. Digital recordings captured each guitarist's session. Following acquisition of the recordings, each note was frequency analyzed and its pitch error recorded. Pitch errors were normalized and summary statistics were calculated. Graphs of summary data by subject and by note were generated for visual analysis.

\section{A. Hardware and Software}

All recordings were made using the same guitar and recording hardware. The guitar was built by the author (Liutaio Mottola "Roberto" model acoustic steel string guitar, scale length $=647.7 \mathrm{~mm}(25.5 \mathrm{in})$ ). The instrument used straight saddle compensation with saddle location calculated using an online calculator (http://www.liutaiomottola.com/formulae/compensation.htm) designed by the author. The guitar was strung with a common string set (D'Addario EJ16), generally referred to as "light gauge" or "twelves". It was set up to typical specifications. String height above the first fret, measured in the conventional manner while fretting at the second fret, was $0.025 \mathrm{~mm}$ (0.001 in). String height of the $\mathrm{G}$ string above the $12^{\text {th }}$ fret was $2.54 \mathrm{~mm}(0.1 \mathrm{in})$. A Peterson TP-3 clip-on pickup was attached to the headstock of the guitar. The pickup was connected to a shop-built unity gain preamplifier. Output of the preamp was connected to the audio input of a Samsung S-3 Android phone. Digital recordings (PCM, $44.1 \mathrm{kHz}$ sampling rate, 16 bit, mono) were made using the Audio Recorder v3.0.43 app (https://gitlab.com/axet/android-audio-recorder) and saved as uncompressed .wav files. Pitch analysis was performed using the Audacity 2.1.3 sound editor and AP Tuner 3.07 (fast display rate, output rounded to 1 cent) on a Windows PC. Prior to use, pitch accuracy of the AP Tuner was verified using generated sound samples with pitches of G3, G3 +1 cent, and G3 -1 cent. Note that note identification here and elsewhere in this article uses the Scientific Pitch Notation format. Pitch error data was stored in an Excel spreadsheet, which was also used for data normalization and the calculation of summary statistics. Graphs were prepared using the Veusz scientific plotting package 2.1.1.

\section{B. Recordings}

Ideally same-note pitch data would be collected from a large group of experienced guitar players and would include a large number of samples at each string/fret location of the instrument. The number of guitar player subjects included in the study was limited to ten as described above, for the sake of expediency. Preliminary testing indicated that pitch variability would not vary much among the six strings of the guitar, so data was collected from the $G$ string only in this experiment. Preliminary testing also indicated that pitch variability at each fret location could be accurately approximated by interpolation from data taken from a limited number of frets. Given these results from preliminary testing and as an attempt to limit subject playing time to maintain subject motivation throughout the experiment, recordings were made only at the first fret $(\mathrm{G} \# 3,195 \mathrm{~Hz})$, fifth fret $(\mathrm{C} 4,261.6 \mathrm{~Hz})$, and twelfth fret $(\mathrm{G} 4,391 \mathrm{~Hz})$ of the G string.

Each player's session was recorded in a single file. Prior to recording, players were presented with a description of the experiment and instructions for what to play. Players were instructed to play a series of notes, each of approximately $1 \mathrm{~s}$ duration and were advised that the test administrator would audibly count each sample and its duration (i.e. "one one thousand, two one thousand, ...") during the recording session. Players were instructed to strive for pitch consistency; and to avoid changes in fretting pressure, string bending, articulation, and vibrato to the extent possible. Players visually monitored the recording level meter on the recording device during playing and were asked to maintain strong signal level but without clipping. To maintain ecological validity, players were instructed to remove both hands from the instrument following each note played. The sequence of notes played during each session included one open string G3 sample, followed by twenty notes each of the above-mentioned fretted notes. Following recording, players were asked to indicate the number of years of guitar playing experience they had. 
Note that no real-time pitch analysis was performed and no electronic real-time pitch feedback was provided to subjects. Providing such high resolution pitch feedback has the potential to result in more accurately pitched notes than would normally be had during conventional playing in a musical context. Preliminary investigation indicated that not providing such feedback would help to maintain ecological validity - subjects could "play to the tuner" if one is available in such an experiment and this can substantially bias results.

\section{Pitch Error Determination}

Pitch errors for each sample were determined using Audacity and AP Tuner. Each subject's recording was opened and the samples were processed sequentially using Audacity. Each sample was selected in the Audacity editor such that the selection did not include the first approximately $50 \mathrm{~ms}$ and also did not include any of the noise following the sample. The initial $\approx 50 \mathrm{~ms}$ was not included to avoid the initial attack portion of the sample, which usually has not settled sufficiently to represent the pitch of the played note. The selected area was played while viewing the output display of the AP Tuner. The initial displayed pitch reading was ignored due to artifacts induced by selection windowing. The pitch error in cents was noted and transcribed to an Excel spreadsheet for further processing.

Due to the approximately $1 \mathrm{~s}$ duration of the samples it was usually the case that indicated pitch changed during the sample. In this case the pitch that persisted longest during the sample was noted. If no single pitch obviously predominated, an average pitch error value was estimated from all values reported by the tuner software. Note an interesting subset pattern which occurred frequently enough that it may warrant additional investigation. In this subset, pitch decreased monotonically over the course of the sample.

\section{Data Normalization and Summary Statistics}

The raw pitch error data were minimally processed in this experiment. Each fretted note datum was first normalized to a perfect tuning condition of the open G3 string. Although the pitch of the open string was regularly tuned between recording sessions, small tuning errors were present in some of the recordings. The first sample in each recording was of the open G3 string. The pitch error value of this sample was subtracted from that of each subsequent sample in the file to normalize all fretted note sample pitch error values, thus allowing meaningful comparison of pitch error ranges across subjects.

Summary statistics including minimum, maximum, mean, median, and standard deviation were calculated for all tuning-normalized fretted note data for each note by subject and also for the entire subject population. Next, an additional data normalization step was performed to offset the tuning-normalized fretted note data for each note by subtracting the population mean value for the note from each datum. This had the effect of offsetting the population mean values to zero error. This data normalization step aids in visual comparison of data for the three notes. It also aids in comparison of the data collected in this study with that found in the referenced works on compensation.

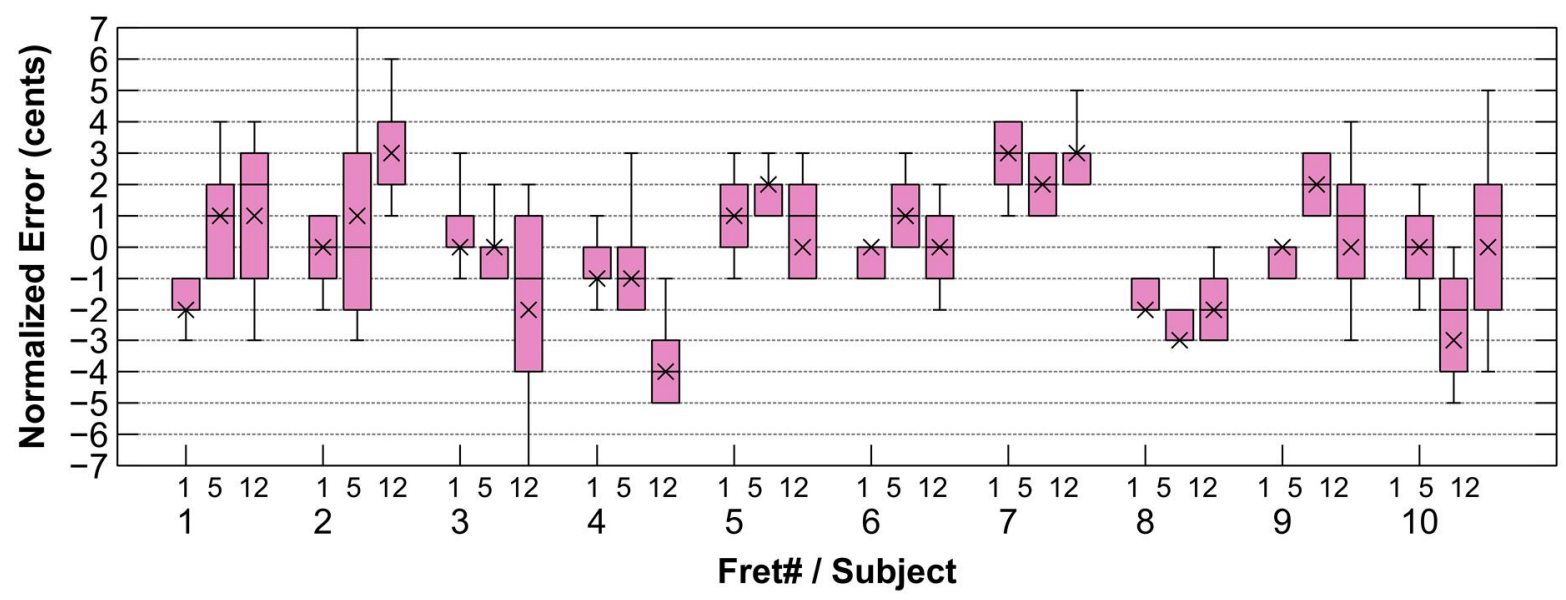

Figure 1 - Pitch variability of three fretted notes presented grouped by subject. Each boxplot represents 20 same-note samples from 1 subject. Whiskers indicate range minimums and maximums. Box ends span one standard deviation from mean. All data have been normalized so mean value for all study samples of each note $(20$ samples per subject $x 10$ subjects $=200)$ is at zero error.

The complete spreadsheet data file including raw and processed pitch error data is named SubjectlntonationData.xlsx and is available as a supplementary file to this article. 


\section{RESULTS}

Results are displayed for visual analysis in figures 1 and $\mathbf{2}$. Figure 1 groups each of the three notes by subject. Figure 2 groups all subjects by note.

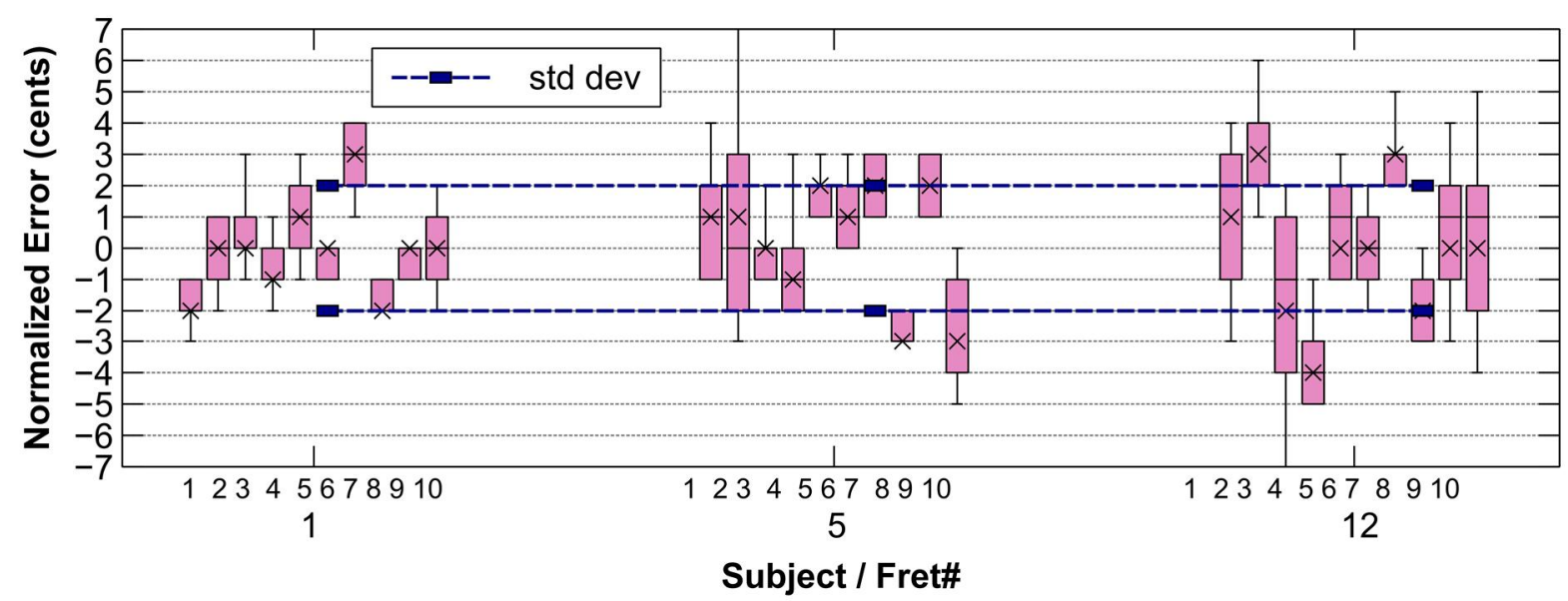

Figure 2 - Pitch variability of three fretted notes presented grouped by note. Each boxplot represents 20 same-note samples from 1 subject. Whiskers indicate range minimums and maximums. Box ends span one standard deviation from mean. All data have been normalized so mean value for all study samples of each note $(20$ samples per subject $\times 10$ subjects $=200)$ is at zero error. Standard deviation of all 200 samples for each note is denoted by blue bars.

\section{DISCUSSION AND CONCLUSION}

Visual analysis indicates substantial differences in both median pitch error values and pitch error ranges across notes for some subjects. Also visible are substantial differences among subjects in both median pitch error values and pitch error ranges for all three notes. Ranges increase strictly monotonically with fret location of the three notes for three subjects. One subject attained ranges of one cent at frets one and five, and a range of three cents at fret twelve. The smallest range attained was one cent and the largest was eleven cents. A general trend can be seen for increased variability of medians and increased ranges with increasing fret location.

Summary statistics indicate that pitch errors for the three notes analyzed varied \pm 2 cents from mean value at one standard deviation, i.e. for $\approx 68 \%$ of samples, and \pm 4 cents for $\approx 95 \%$ of samples. Reasonable assumptions about the representativeness of study subjects and interpolation of results to additional fretted notes of the string analyzed in the study may indicate the results can be generalized to most guitar players and to other fretted notes as well. Although the playing conditions of the experiment are highly contrived they were designed to represent a feasible musical context in which players can attain the best possible same-fretted-note intonation consistency. Future research exploring intonation consistency in more typical musical contexts may be useful.

\section{ACKNOWLEDGMENTS}

Thanks to the guitarists that took part in this study and to the New England Luthiers group which provided access to these subjects at one of its regular meetings. Thanks to Sjaak Elmendorp for useful comments on an early draft of this article. Thanks to Christine King for proofreading and editing work. This paper and the research it describes are substantially improved by these efforts.

\section{BIBLIOGRAPHY}

1. Bartolini, W. and Bartolini, P. (1982). Experimental Studies of the Acoustics of Classic and Flamenco Guitars. Journal of Guitar Acoustics 6, 74

2. Gilbert, J. and Gilbert, W. (1984). Intonation and Fret Placement. Soundboard 26-27

3. Byers, G. (2006). Classical Guitar Intonation. American Lutherie \#47 p.34 
4. Doolin, M. (2007). Intonation in the Real World. American Lutherie \#92 p.26

5. Gore, T. and Gillet, G. (2011) Contemporary Acoustic Guitar Design and Build

6. Magliari, G. and MacRostie, D. (2013). Beyond the Rule of 18: Intonation for the 21st Century. American Lutherie \#116 p.6

7. Varieschi, G. U. and Gower, C. M. (2010). Intonation and compensation of fretted string instruments. American Journal of Physics, 78(1), 47-55.

8. Shower, E. G. and Biddulph, R. (1931). Differential pitch sensitivity of the ear. The Journal of the Acoustical Society of America, 3(2A), 275-287.

9. Mottola, R.M. (2017, October 21). Blind Listening Evaluation of Steel String Acoustic Guitar Compensation Strategies. OSFPreprints. Retrieved from https://osf.io/bqzf4/

10. Mottola, R. M. (2014). Guitar Fretboard Camber and Action in the Context of String Bending. Savart Journal, 1(4). 